Abstract P73 Table 1 Demographics of study population $(n=325)$

\begin{tabular}{ll}
\hline Age (mean SD) & $49(14)$ \\
\hline Gender & $48 \%$ female \\
BMI & $35.9(9.3)$ \\
AHI (valid sleep study at time of analysis in 282) & $18.7(19.6)$ \\
ESS & 10.5 \\
Diagnostic breakdown & Mild OSA (21\%); Mod OSA (18\%); \\
& Severe OSA (22\%); Other (39\%) \\
\hline
\end{tabular}

the 'clinical dashboard' used to highlight difficult cases for a weekly MDT. The CDSS generates automated clinical letters for each review thus greatly reducing secretarial time/costs for the service as no typing is required.

Conclusion The implementation of an intelligent Computer Guided Consultation system has resulted in pathway transformation enabling scarce Consultant resource to be channelled to where it is most required and enhancing service capacity, efficiency and patient safety. Adopting the system results in multi-level health economic benefits and facilitates greater service oversight.

Please refer to page A192 for declarations of interest related to this abstract.

\section{P74 DIGITAL TRANSFORMATION - THE BEATING HEART OF A MODERN COPD SERVICE}

S Gompertz, R Wagstaff, S Rees, R Colclough. Queen Elizabeth Hospital Birmingham, University Hospitals NHS Foundation Trust, Birmingham, UK

\subsection{6/thorax-2021-BTSabstracts. 184}

Introduction and Objectives We commenced an early supported discharge (ESD) service for COPD patients in March 2020. We describe the number of patients presenting to our hospital since then, our virtual in-patient multi-disciplinary team
(MDT) ward-round and the benefits that this has brought to our COPD service.

Methods A pilot ESD service developed jointly between the acute hospital and community respiratory team uses co-prescription of prednisolone $\geq 30 \mathrm{mg}$ AND regular nebulised bronchodilators in our electronic prescribing information and communication system (PICS) to send an alert to the Blackberries of our specialist respiratory nursing/physiotherapy team (RST). They conduct a remote assessment to identify those patients with COPD, and attend the bedside to complete a comprehensive COPD discharge bundle. They also provide clinical advice to the in-patient medical team on prescribing, inhaler device selection, target saturations, etc, calculating an initial DECAF score, and using this with a respiratory consultant's support to identify patients for ESD.

Results and Service Description Completion of the COPD bundle generates an in-patient virtual COPD ward allowing the respiratory consultant to conduct a virtual ward-round each morning. DECAF score is finalised, suitable patients are identified for ESD, and advice about treatment, investigation and follow up are written in PICS; changes in prescriptions are made where required. The consultant and RST communicate by email and/or phone about the management of the patients providing a virtual in-patient MDT ward-round.

The virtual ward is also used to: support door-to-mask time quality improvement; to optimise run chart data entry for the National Asthma and COPD Audit Programme (NACAP; smoking cessation, oxygen prescription, spirometry results) and to populate our NACAP returns; to optimise the remainder of the COPD care bundle including antibiotic prescription, thromboprophylaxis, steroid and nebulised bronchodilator prescription, with advice about respiratory failure and ward destination using our COPD care bundle mnemonic (AECOPD $-\mathrm{R}^{2} \mathrm{D}^{2}$ ). The COPD bundle is sent automatically to the GP portal when the patient is discharged from hospital.

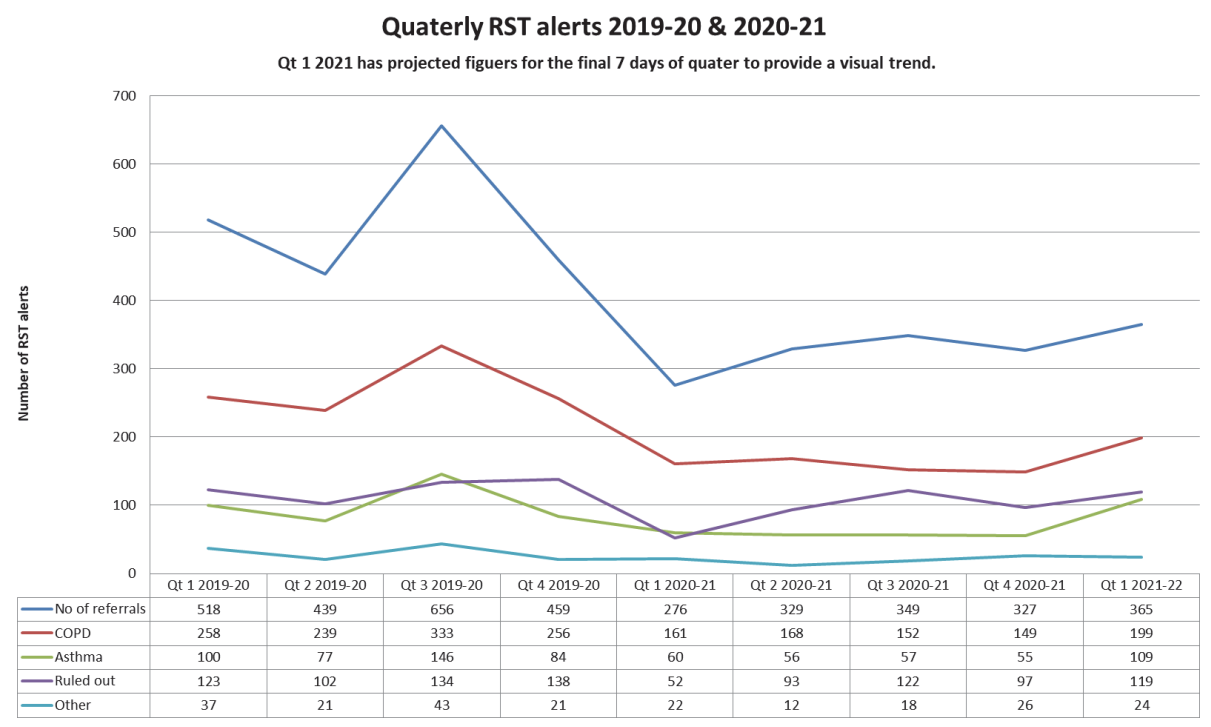

Abstract P74 Figure 1 Total no. of alerts (upper, blue line) and COPD alerts (second uppermost, red line) sent to RST over time - the impact of COVID on acute admissions can clearly be seen by the marked reduction in Q1 2020-2021 which also corresponds with when the pilot service started

(Asthma - RST also review patients with asthma. Ruled out = on clinical review patient had neither COPD nor asthma; Other = not reviewed by RST (short stay, self discharge or died prior to review)). 
Conclusion Our ESD pilot has allowed us to develop a COPD service with IT at its heart. This has led to many improvements including a novel daily in-patient virtual MDT wardround.

Please refer to page A192 for declarations of interest related to this abstract.

\section{P75 A COMPARISON OF TELEPHONE VERSUS FACE-TO-FACE CONSULTATIONS WHEN COMMENCING CPAP THERAPY FOR OBSTRUCTIVE SLEEP APNOEA}

MV Foster, MTC Thomas, S Mitchell, T McKown, SE Craig. Liverpool University Hospitals NHS Foundation Trust, Liverpool, UK

\subsection{6/thorax-2021-BTSabstracts. 185}

Background The Covid-19 pandemic has led to the cancellation of a large majority of face-to-face clinic appointments across the NHS, prompting a shift towards telephone reviews. Although perceived as being inferior in some circumstances where direct patient contact is vital for picking up on physical signs and non-verbal cues, for many areas of medicine this could increase efficiency and minimise disruption to patient's lives whilst delivering comparable outcomes. ${ }^{1}$ In this study, we compared CPAP compliance in patients with obstructive sleep apnoea reviewed via telephone with those seen in face-to-face clinics.

Methods Forty patients who attended for CPAP set up were allocated to receive either telephone or face-to-face compliance appointments with twenty patients in each cohort. They were reviewed at 48 hours post CPAP initiation, seven days, one month and six months. At each review their apnoea-hypopnoea index (AHI) and compliance percentage was downloaded. An unpaired T-test was used to compare the two groups.

Results There were 14 males and 6 females in the telephone cohort versus 15 males and 5 females in the face-to-face cohort. The telephone group were aged 32-69 (median age 53) whereas the face-to-face group were aged 30-66 (median age 46).

The mean (SD) compliance at six months in the telephone cohort was $43.8 \%(36.7 \%)$, compared with $45.6 \%(38.5 \%)$ in the group who had had face-to-face reviews $(p=0.869)$. The

Abstract P75 Table 1 The mean (standard deviation) of CPAP compliance and apnoea-hypopnoea index in telephone and face-toface groups at various time points

\begin{tabular}{lllllll}
\hline & Compliance & \multicolumn{5}{c}{ AHI } \\
\hline & Telephone & Face-to-Face & p- \\
value & Telephone & $\begin{array}{l}\text { Face-to- } \\
\text { Face }\end{array}$ & $\begin{array}{l}\text { p- } \\
\text { value }\end{array}$ \\
\hline Initial & n/a & n/a & n/a & $38.6(19.3)$ & $39.5(24.3)$ & n/a \\
48 Hours & $71.5 \%$ & $61.7 \%$ & 0.154 & $3.8(3.6)$ & $6.3(7.8)$ & 0.007 \\
& $(37.0 \%)$ & $(44.6 \%)$ & & & & \\
7 Days & $58.0 \%$ & $57.2 \%$ & 0.840 & $4.7(4.5)$ & $5.1(4.6)$ & 0.661 \\
& $(40.5 \%)$ & $(40.3 \%)$ & & & & \\
1 Month & $54.8 \%$ & $47.5 \%$ & 0.457 & $3.9(3.0)$ & $5.9(4.8)$ & 0.092 \\
& $(36.2 \%)$ & $(38.4 \%)$ & & & & \\
6 & $43.8 \%$ & $45.6 \%$ & 0.869 & $3.5(2.8)$ & $5.6(4.5)$ & 0.057 \\
Months & $(36.7 \%)$ & $(38.5 \%)$ & & & & \\
\hline
\end{tabular}

mean (SD) AHI at six months in the telephone cohort was 3.5 (2.8), compared with 5.6 (4.5) in the face-to-face group $(\mathrm{p}=0.057)$. The results show no significant difference between the two groups.

Conclusion Conducting telephone consultations with patients who are being set up on CPAP was shown to be non-inferior to having face-to-face reviews. This study supports the idea that telephone reviews are a safe alternative to traditional face-to-face clinics.

\section{REFERENCE}

1. Car J, Koh GCH, Foong PS, Wang CJ. Video consultations in primary and specialist care during the covid-19 pandemic and beyond. BMJ 2020;371:m3945. doi: https://doi.org/10.1136/bmj.m3945

\section{P76 IMPROVING COMMUNITY-BASED CARE USING ONLINE COMMUNICATION PORTAL FOR PATIENTS WITH AN INDWELLING PLEURAL CATHETER}

J Liang, K Ur Rehman, P Sivakumar. Guys and St Thomas' NHS Foundation Trust, London, UK

\subsection{6/thorax-2021-BTSabstracts. 186}

Introduction and Objectives Indwelling pleural catheter (IPC) manufacturers offer an online portal to facilitate communication between secondary care services and the district nurses (DNs).

We aimed to characterise the experiences of patients and DNs of community-based IPC care that is supported by an online communication portal.

Methods A manufacturer-based online portal was adopted by our service in February 2021 with data prospectively collected on IPC care. Qualitative feedback was sought from patients and district nursing teams during follow-up.

Results 13 eligible patients underwent IPC insertion between February 2021 and June 2021. 100\% (13/13) patients agreed to be enrolled onto the portal.

Seven issues affected six patients during follow up, four of which were addressed in the community.

Four phone calls were documented regarding bottle shortages. Via the portal, overnight delivery of bottles to patient's address was organised on all occasions. DNs were also trained on fast-track bottle requisition.

In three patients, sutures were not removed as instructed, which was identified at planned hospital follow up. Via the portal, the manufacturer contacted DNs to organise refresher training. Two DN teams accessed this course and reported benefits, finding it particularly useful for their novice practitioners. The training covered all aspects of the product, drainage procedure, ordering process and troubleshooting.

All patients reported satisfaction with their communitybased care during the follow up period. The four patients with bottle issues were grateful for a prompt resolution without the need to attend the hospital.

One patient reported a 'sense of confidence in the care being provided in the community.'

Conclusion The online portal ensures seamless communication between a secondary care pleural service and the local district nursing teams, avoiding the need for healthcare attendance through prompt resolution of community-based issues. It also facilitates the provision of community-based training. The partnership between these teams ensures better care quality, clinical outcome and support for patients. 